\title{
Experimentally based simulations on modulated lidar for shallow underwater target detection and localization
}

\author{
Vincent Jezequel $^{* a}$, Frederic Audo $^{\mathrm{b}}$, Fabrice Pellen $^{\mathrm{a}}$, Bernard Le Jeune $^{\mathrm{a}}$ \\ ${ }^{a}$ Université Européenne de Bretagne, Université de Bretagne Occidentale, Laboratoire de \\ Spectrométrie et Optique Laser (EA 938), 6 avenue Le Gorgeu C.S. 93837, 29238 Brest Cedex, \\ France ; \\ ${ }^{\mathrm{b}}$ Ecole Nationale d'Ingénieurs de Brest, Laboratoire RESO (EA3380), C.S. 73862, 29238 Brest \\ Cedex 3, France.
}

\begin{abstract}
Light detection and ranging (LIDAR) is currently used for bathymetric measurement or underwater target detection. A new underwater-target detection scheme named modulated lidar was recently proposed. The study reported here deals with optimization of the modulation process to be applied under such detection conditions. A theoretical model was extracted from available experimental results by deconvolution and further used to simulate realistic backscattered signals for the development of a new modulation scheme. Then, an optimum set of amplitude modulation code parameters was obtained by maximizing the target signal-to-noise ratio. This paper will highlight some particularly promising waveform configurations.
\end{abstract}

Keywords: Laser radar, shallow underwater-target detection and ranging, modulated lidar, radiofrequency amplitude modulation, narrow band filtering

\section{INTRODUCTION}

LIDAR is currently employed for bathymetric measurement and/or underwater target detection. An optical source commonly used for a conventional lidar system is a pulsed Nd:YAG laser frequency-doubled at $532 \mathrm{~nm}$. Indeed, absorption in the seawater is minimized by the blue-green wavelength ${ }^{1}$. Nevertheless, the volume backscattering in the water column can be considered as a deterministic noise since it superimposes on the target echo. By severely limiting detection performances, this backscattering clutter may cause numerous false alarms, especially for shallow underwater target and/or when targets have a weak albedo.

According to previous studies, the seawater has a low-pass transfer function in a backscattering configuration with a cut-off frequency up to several $\mathrm{MHz}^{2}$. As the target return is less frequency-dependent, the use of a radiofrequencymodulated laser source along with a narrow band filtering at the detection drastically reduces the backscattering clutter ${ }^{3,4}$, but has no effect on the target return; the modulation frequency of the source must be clearly above the cut-off frequency of the propagating medium. This high-frequency modulated-lidar detection scheme was initially proposed by Mullen $e t$ $a l^{5}$ then developed by Pellen et al. ${ }^{6}$ within our laboratory through experiments carried out in a water tank $k^{2,6}$. The amplitude-modulated laser pulse was obtained through use of an optical delay line system placed after a 100-ps-pulse $\mathrm{Nd}$ :YAG laser frequency-doubled at $532 \mathrm{~nm}$. At the required frequency $(1.5 \mathrm{GHz})$, adjustment of both temporal delay and intensity ratio of each arm gives a set of 4 identical pulses of equal-amplitude and -periodicity. By producing conclusive experimental results, this set-up permitted great enhancement of the target detection contrast ${ }^{6}$.

The study presented here was aimed at optimizing the modulation code. In order to enhance detection, e.g. to increase both the target signal-to-noise ratio and the signal-to-volume-backscattering-noise ratio, we devised a new modulation scheme for the emitted lidar pulse: it consists in the use of several equally-spaced 100-ps pulses with decreasing amplitudes and easily adjustable modulation frequency.

*vincent.jezequel@univ-brest.fr; phone: 33 (0)2.98.01.82.78 
About signal processing, as this new modulation is more frequency-efficient, it is expected to reduce more effectively the background noise with no loss of temporal shortness, which is crucial in target ranging. With this new modulation, the emitted signal depends mainly on three parameters: the modulation frequency, the number of emitted pulses and the decreasing rate. To gain more insight into their impact, simulations were run prior to the development of the experimental set-up. A theoretical model was, thus, extracted by deconvolution from available experimental results ${ }^{6}$ and further used to simulate realistic backscattered signals for different input signals. This model took into account the seawater backscattering, the target echo and the set-up-induced spurious echoes. Then an optimum set of parameters was extracted for the amplitude modulation code by maximizing both the target signal-to-noise ratio and signal-to-volumebackscattering-noise ratio.

The present paper is organized as follows: Section 2 introduces the methodology used to extract, by deconvolution, the theoretical model from available experimental data. After description of the experimental set-up, the signal processing techniques in use are presented together with the performance assessment criteria. Section 3 describes the new modulation scheme as well as the simulations results. The conclusion is drawn in Section 4.

\section{METHODOLOGY}

\subsection{Theory}

A single scattering treatment of the backscattered light pulses in the water volume shows that time spreading for a homogenous medium can be expressed by ${ }^{7,8}$ :

$$
B(t)=W(t)^{*} E(t)
$$

where $*$ denotes the convolution operator, $E(t)$ is the reference incident light pulse, and

$$
W(t)=\frac{\mathrm{v}}{2 n} \beta(180) \exp \left(-\frac{c \mathrm{v} t}{n}\right) \Omega(t) .
$$

is the water backscattering impulse response, $c$ is the medium extinction coefficient, $\beta(180)$ is the backscattered diffusion coefficient, $\mathrm{v} / n$ is the celerity of the electromagnetic wave in the medium, $n$ is the refractive index, and $\Omega(\mathrm{t})$ is a fieldof-view correction. This correction takes into account the geometric parameters of the lidar system as follows ${ }^{7,8}$ :

$$
\Omega(t)=\frac{1}{\left(n \ell+\frac{\mathrm{v} t}{2 n}\right)^{2}} .
$$

where $\ell$ is the distance between the detector and the scattering medium.

Eqs. (2) and (3) may become more complicated due to the possible presence of a multiple-scattered component. However, simulations run on using a semi-analytical Monte Carlo treatment as described by Poole et al. ${ }^{9}$ showed us that, under our experimental conditions, this contribution was limited because of both the small numerical aperture of the detection system and the relatively low scattering coefficients $\left(c<1 \mathrm{~m}^{-1}\right)$.

\subsection{Experimental set-up}

Figure 1 describes the experimental set-up used in the past within our laboratory. The modulated signal is produced by an optical delay line modulator ${ }^{6}$ (Figure 2) placed after a 100-ps pulse produced by a Nd:YAG laser frequency-doubled at $532 \mathrm{~nm}$. The adjustment of both the delay and intensity ratio of each arm gives a train of 4 identical pulses equalamplitude and -periodicity at the chosen modulation frequency. This 4-pulse train forms a 3-ns modulated pulse. 


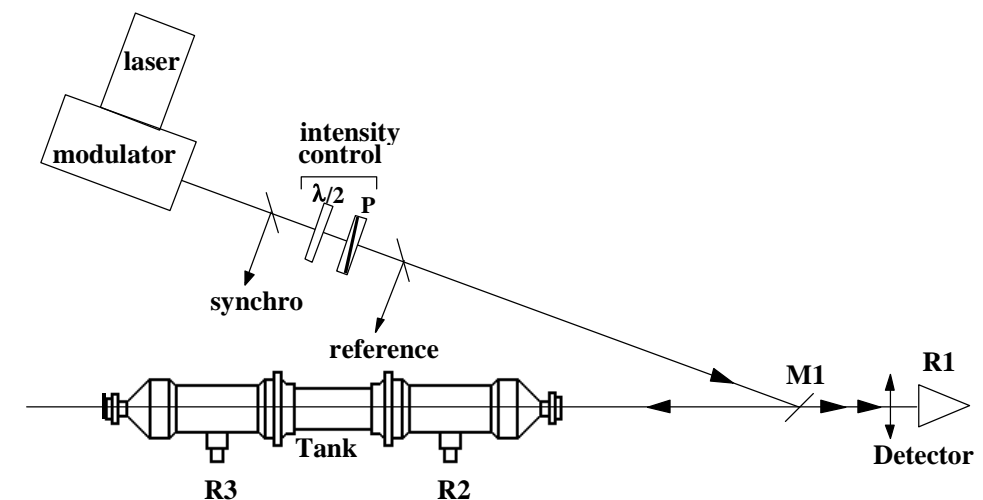

Figure 1. Experimental set-up: frequency-doubled Nd:YAG laser with a 3-ns amplitude-modulated pulse.

R1: detector for measuring the backscattered signal, R2 and R3: positions of the lateral detector for scattering coefficient measurements, M1: $10 \mathrm{~mm}$ diameter mirror, 5-m-long experimental tank (diameter $30 \mathrm{~cm}$ ), filled with filtered tap water and calibrated clay powder particles.

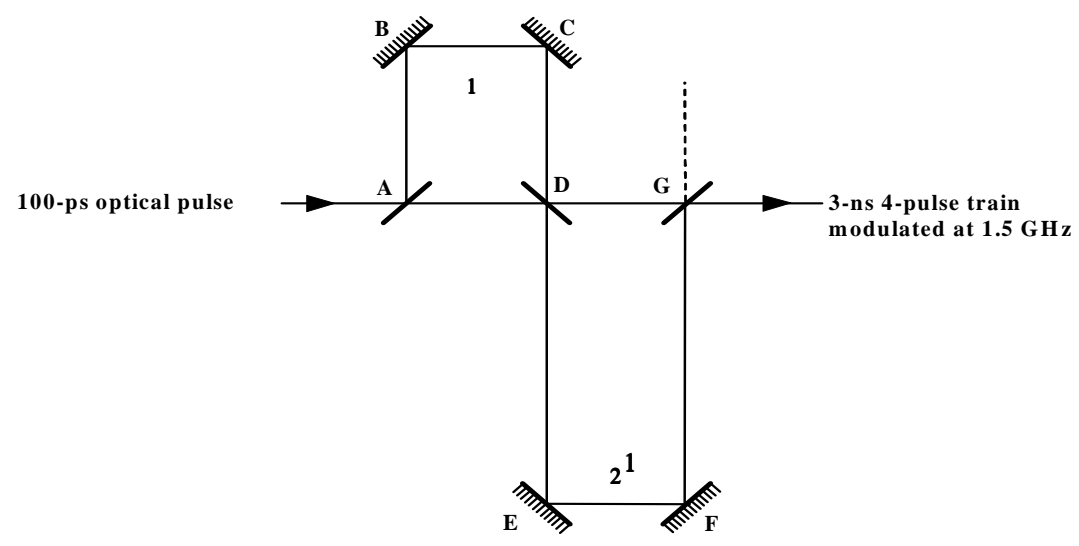

Figure 2. Optical delay line modulator for the generation of a 3-ns 4-pulse train modulated at $1.5 \mathrm{GHz}$. A, D and G: 50/50 beam splitters; B, C, E and F: mirrors.

Figure 3(a) shows an experimental plot of the 100-ps optical pulse recorded with a microchannel plate photomultiplier tube associated with a high bandwidth analyzer (Tektronix CSA 803) whose frequency response is $3 \mathrm{GHz}$ at $-3 \mathrm{~dB}$. One should note oscillations at high frequency, i.e. $1.11 \mathrm{GHz}$, just behind the pulse. They likely result from a mismatched impedance along the measurement chain. However, they were kept to make sure that the rising times were short. Figure 3(b) presents the optical signal obtained at the delay line modulator output and recorded by the same detection chain. The time period of this output signal is $666 \mathrm{ps}$, which corresponded to a 1.5-GHz modulation frequency. The modulation efficiency, $m$, of the delay line system is only $58 \%$ because of the high frequency oscillations noticed along the detection chain. This 3-ns modulated pulse was further used to assess the modulated lidar capability in underwater target detection. 


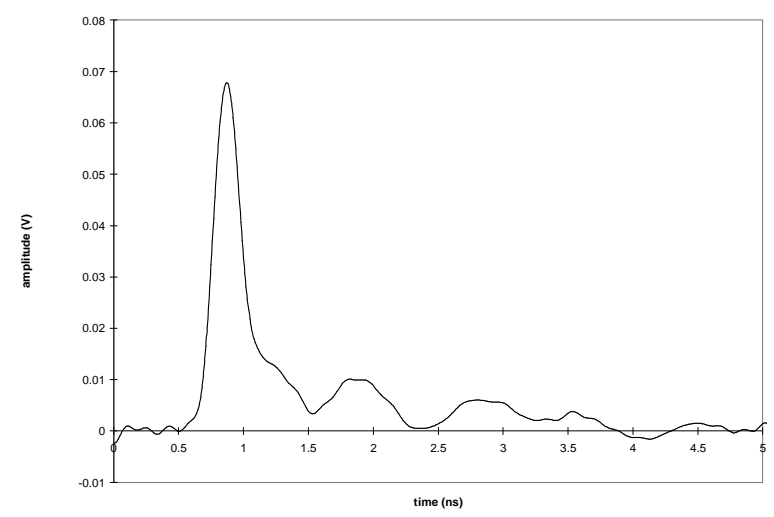

(a)

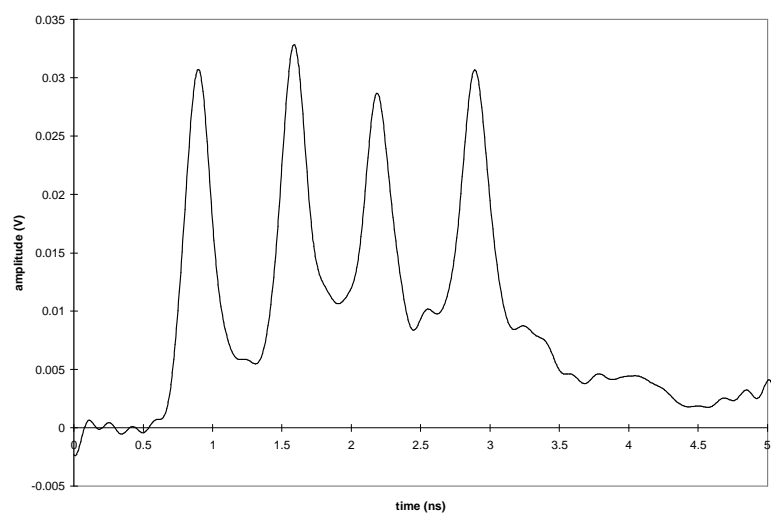

(b)

Figure 3. (a) 100-ps optical pulse generated by the Nd:YAG laser; (b) 3-ns modulated pulse.

Figure 4 displays the experimental shape of the backscattered signal $S_{e}(t)$ recorded in the R1 direction and issued from a target immersed in water at a 5-m depth and for a medium extinction coefficient of $c=0.67 \mathrm{~m}^{-1}$. The 7-cm-diameter target used was the tank output window.

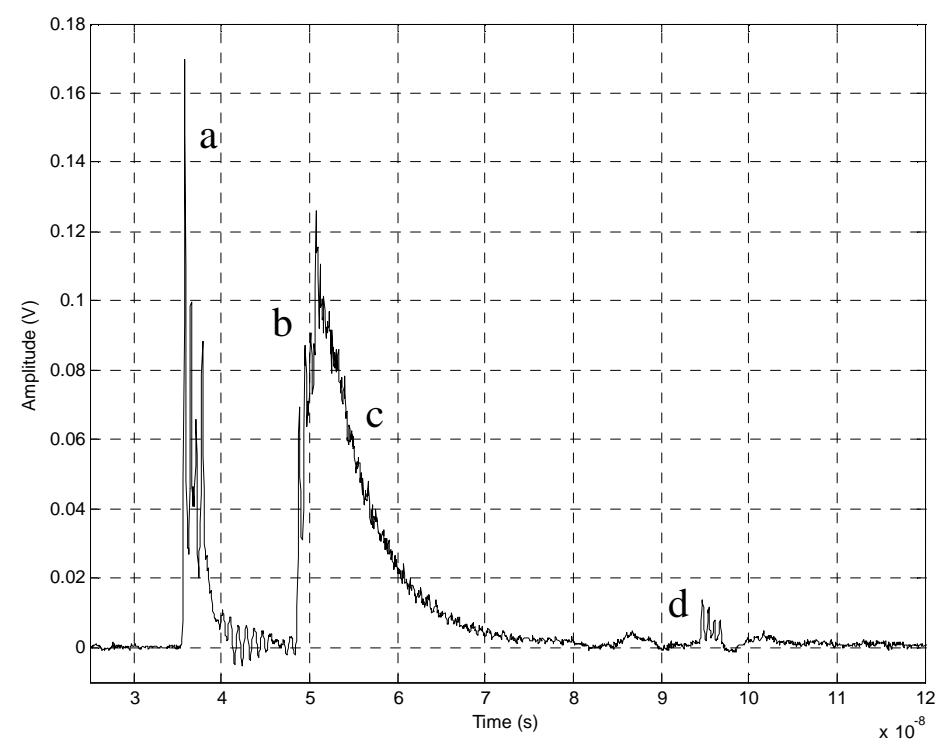

Figure 4. Experimental backscattered signal $S_{e}(t)$ from a 5-m deep underwater target $\left(c=0.67 \mathrm{~m}^{-1}\right)$.

(a): diffusion effect on the small mirror M1, (b): echo due to the diffusion on the entrance window, (c): volume backscattering signal, (d): reflection on the target.

One should note that, as expected from the theoretical study ${ }^{5,6}$, the medium backscattering signal (c) seems to not contain the modulated signal, whereas the modulated code is present in the target signal (d) as well as in the set-up-induced echoes (a) and (b). So, a signal processing consisting in a narrow band pass detection centered at the modulation frequency $(1.5 \mathrm{GHz}$ in the present case) must drastically reduce the backscattering clutter, but not affect the target signal. The target signal-to-volume-backscattering-noise ratio should be consequently greatly enhanced by the processing. 


\subsection{Theoretical model deconvolution}

The theoretical model needed for simulations was extracted by deconvolution of the backscattered experimental signal $S_{e}(t)$ shown in Figure 4. Figure 5 presents an experimental plot of the 3-ns modulated optical pulse recorded with this photomultiplier and the high bandwidth analyzer with a diffuse target placed at the entrance window of the tank. Let us denote by $E(t)$ this signal considered hereafter as the reference signal and used in the deconvolution process. The four main echoes (a, b, c and d on Figure 4) visible on the experimental backscattered signal $S_{e}(t)$ are easily modeled and expressed as follows:
(a) the diffusion effect on the small mirror, $\mathrm{M}_{1}: a_{1} E\left(t-\tau_{1}\right)$
(b) echo due to the diffusion on the entrance window: $a_{2} E\left(t-\tau_{2}\right)$
(c) the volume backscattering signal: $a_{3} E\left(t-\tau_{3}\right) * W(t)$
(d) the reflection on the bottom window: $a_{4} E\left(t-\tau_{4}\right)$

where $W(t)$ is the water backscattering impulse response described by Eq. (2).

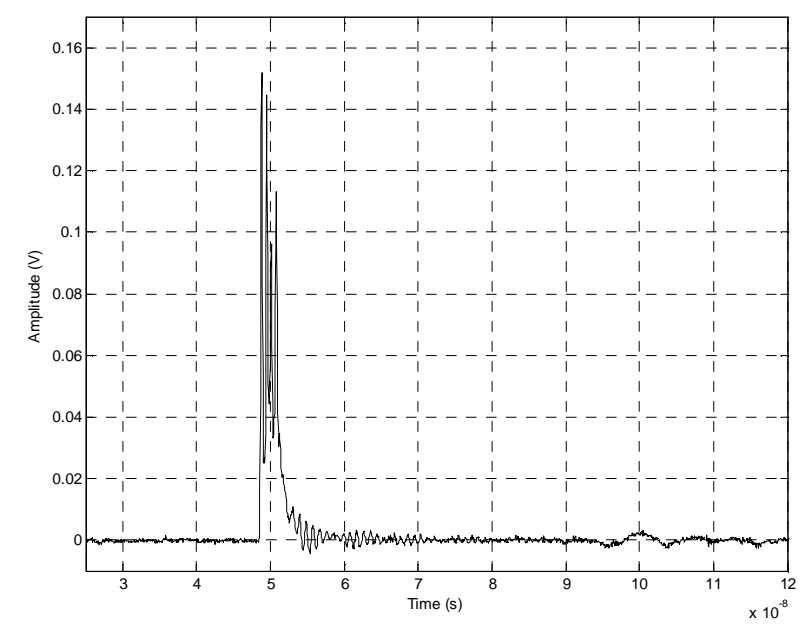

Figure 5. Reference signal $E(t)$ : backscattered signal from a diffusing target placed at the tank entrance.

As the reference signal and the backscattered one are both experimental signals, they are corrupted by various noises (low and white frequency noises). This led us to extract the ocean water impulse response by a model fitting method. The backscattered signal model, $S_{m}(t)$, can thus be expressed as follows:

$$
S_{m}(t)=a_{1} E\left(t-\tau_{1}\right)+a_{2} E\left(t-\tau_{2}\right)+a_{3} E\left(t-\tau_{3}\right) * W(t)+a_{4} E\left(t-\tau_{4}\right) .
$$

where the parameters in $S_{m}(t)$ are adjusted by a least squares method ( $\chi^{2}$ minimization) to fit the experimental backscattered signal, $S_{e}(t)$.

$$
\chi^{2}=\sum\left(S_{m}(t)-S_{e}(t)\right)^{2}
$$

One should, however, note that several parameters are a priori known from the experimental set-up geometry, and the temporal localization of the different components $\left(\tau_{1}, \tau_{2}, \tau_{3}\right.$ and $\left.\tau_{4}\right)$ is among them. The number of parameters to be adjusted is therefore reduced. Figure 6 compares the measured backscattering response, $S_{e}(t)$, with the fitted backscattering response, $S_{m}(t)$, for $c=0.67 \mathrm{~m}^{-1}$ and shows a very good agreement between both signals. 


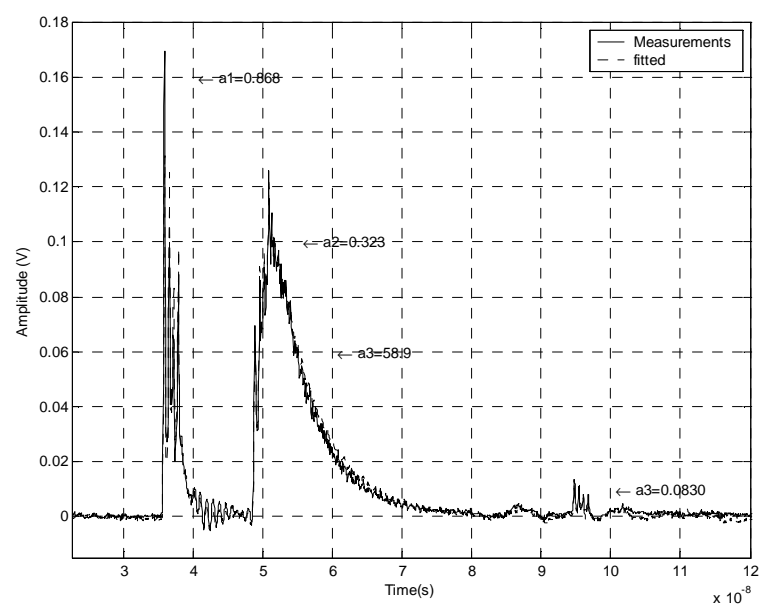

(a)

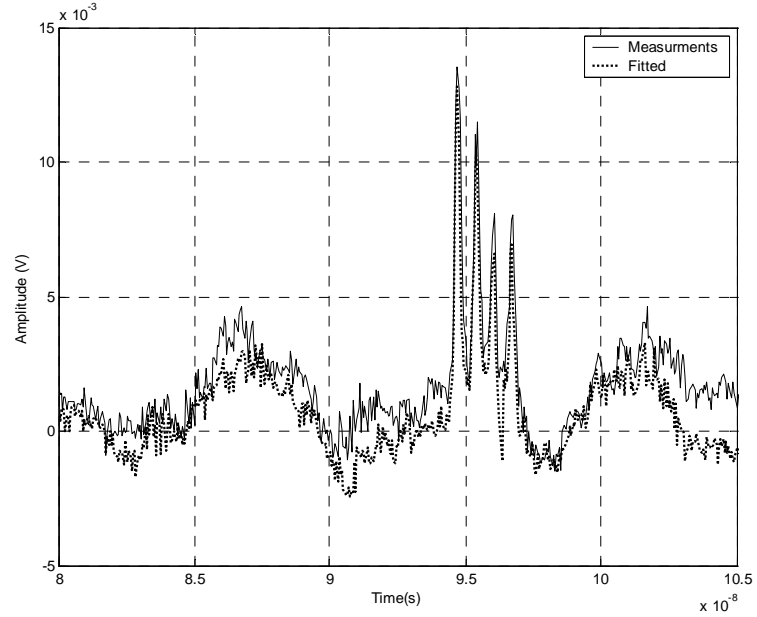

(b)

Figure 6. Comparison between the measured $S_{e}(t)$ and fitted backscattering responses $S_{m}(t)$ for $c=0.67 \mathrm{~m}^{-1}$. (a) full signals; (b) focus on target echo

Let us denote by $M(t)$, the realistic theoretical model of the impulse response given by the experimental set-up (Figure 1). It is calculated from the set of parameters obtained above by the fitting method. Its expression is given by Eq. (7):

$$
M(t)=a_{1} \delta\left(t-\tau_{1}\right)+a_{2} \delta\left(t-\tau_{2}\right)+a_{3} \delta\left(t-\tau_{3}\right) * W(t)+a_{4} \delta\left(t-\tau_{4}\right) .
$$

Then, the simulated backscattered responses, $S_{s}(t)$, for various input reference signals, $E(t)$, is obtained by a simple convolution product as follows:

$$
S_{s}(t)=M(t) * E(t) .
$$

But, the fact that any experimental measurement is corrupted by noise leds us to estimate from experimental data that a $5 \%$ multiplicative noise with a zero mean and an additive gaussian white noise with a standard deviation of $5.10^{-4} \mathrm{~V}$ had to be added to the simulated backscattered signal.

This model, $M(t)$, will be used later (Section 3) to simulate backscattered responses for various input signals.

\subsection{Backscattered signal processing}

To reveal the target reflection hidden in the volume backscattering, a narrow band filtering was placed at the receiver end to filter out the low-frequency part of the return signal and the white noise. Various narrow-band filtering processes are available. The one chosen in this study is a numerical narrow band filtering of the return signal through computation of its cross-correlation with the centered reference signal. Indeed, in this case, the centered reference signal can be considered as a matched-filter. As the medium has a low-pass frequency response, it is essential for the reference signal to not have low-pass component (i.e. to be centered). The reference signal mean-value can be suppressed by different methods. The expected complexity of the simulated reference signals to be further investigated (Section 3 ) drove us to suppress the low-pass component of the reference signal by a high-pass filtering.

Figure 7(a) illustrates the frequency response of the normalized matched-filter issued from the centered reference signal, whereas Figure 7(b) represents the centered reference signal, $E_{c}(t)$ used to compute the cross-corelation. 

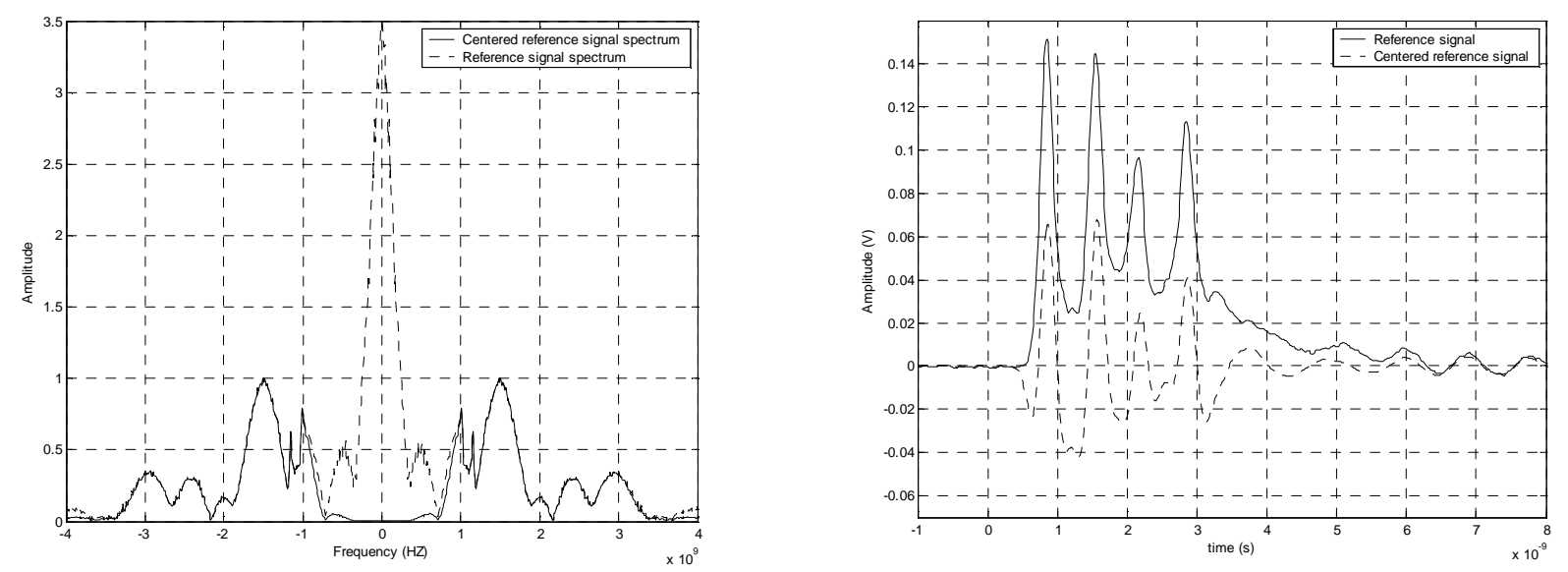

Figure 7. Centered reference signal, $E_{c}(t)$, used as matched filter for backscattered signal processing.

(a) frequency domain representation (Fourier transform of $E_{c}(t)$ ); (b) time domain representation

Let us recall that the cross-correlation of the signals, $f(t)$ and $g(t)$, is defined by:

$$
C_{f g}(t)=\int_{R} f(t) \cdot g^{*}(t-\tau) d t=f(t) * g^{*}(-t)
$$

where $*$ denotes the convolution operator, and $g *(t)$ is the complex conjugate of $g(t)$. The cross-correlation is representative of the similarity between two signals. The comparison of one signal section to another one shifted in time allows one to either detect hidden periodicity or reveal a noise-embedded signal. For two uncorrelated signals, i.e. signal and noise, their cross-correlation is a constant value equal to the product of their respective average values. As the reference signal mean value is equal to zero, the cross-correlation with the volume backscattering component is very weak, which enhances the target contrast.

The cross-correlation of the experimental backscattered signal, $S_{e}(t)$, with the centered reference signal, $E_{c}(t)$, is presented on Figure 8.

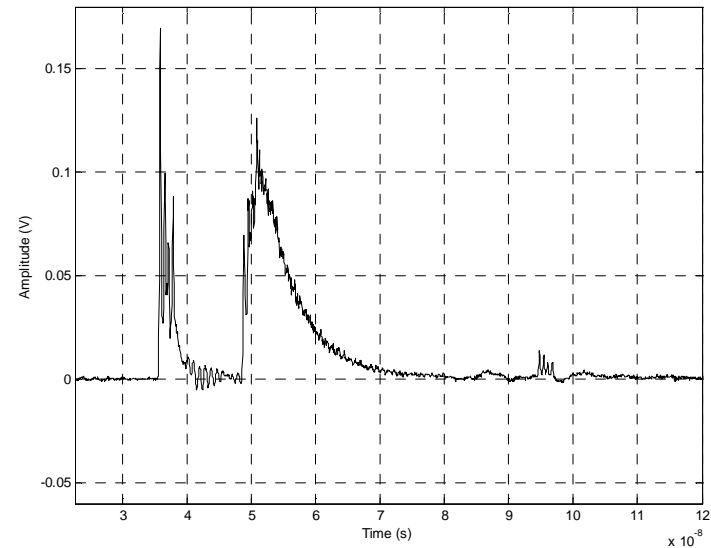

(a)

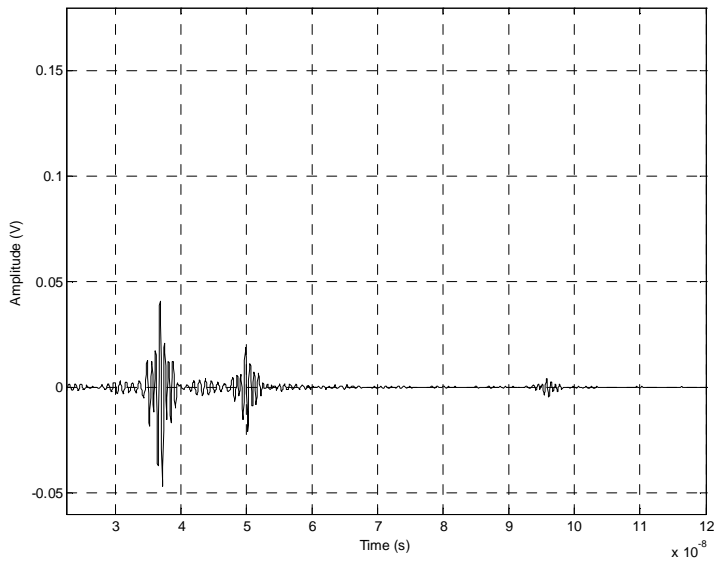

(b)

Figure 8. Experimental signal matched-filtering $\left(c=0.67 \mathrm{~m}^{-1}\right.$ and a 5-m deep target).

(a) experimental signal; (b) cross-correlation result. 
As expected from theory, a comparison of Figures 8(a) and 8(b) shows a drastic reduction of the water volume backscattering by the modulation approach and associated signal processing.

\subsection{Performance assessment}

As the signal is corrupted by, at least, two kinds of noise (deterministic low-frequency backscattering noise and unpredictable white noise), the performances of the simulated modulation scheme under study have to be quantified. The assessment criteria chosen in this study are:

i) The signal-to-noise ratio $(S N R)$, which compares the target echo level to the background white noise level. It is defined as the target signal energy-to-the noise energy ratio:

$$
S N R=\frac{E_{\text {target } \cdot \text { signal }}}{E_{\text {noise }}}
$$

One should note that the raw noise energy and raw target signal energy were both set at constant values whatever the simulations. Moreover, the noise energy was estimated within the first $15 \mathrm{~ns}$ of the backscatered signal.

ii) The signal-to-volume-backscattering-noise ratio $(S V B N R)$, which compares the target echo level to the water backscatered signal level. It is defined as the target signal energy-to-water backscatering energy ratio:

$$
S V B N R=\frac{E_{\text {target } \cdot \text { signal }}}{E_{\text {volume.backscaterring.noise }}} .
$$

iii) The Contrast, which quantifies to which extent the target signal can be clearly distinguished from background noise. It is defined as:

$$
C=\frac{A_{\max }-\sigma_{\text {noise }}}{A_{\max }+\sigma_{\text {noise }}} .
$$

where $A_{\max }$ is the maximum amplitude of the target echo, and $\sigma_{\text {noise }}$ is the standard deviation of the noise.

In conclusion, the detection of underwater targets was greatly enhanced by using a radiofrequency-modulated laser source. Indeed, in addition to the classical range gating localization, the association of a pulse-modulated lidar with a numerical matched-filter permits the detection of a target embedded in the backscattering and acquisition clutter thanks to theirs drastic reduction by the modulation-filtering coupling.

Furthermore, the very good agreement found between the measured backscattering response and the fitted backscattering response, $S_{e}(t)$ and $S_{s}(t)$ respectively, allows us to investigate other modulation schemes through simulations presented in the next section.

\section{SIMULATIONS}

The simulations presented in this section relied on the use of the theoretical model extracted, by deconvolution, from experimental data. Based on this model, realistic backscattered signals were simulated for different input signals. These signals under study, $E(t)$, depend on the modulation frequency, the number of pulses and the decreasing rate.

\subsection{Modulation frequency}

By generating a long tail after the pulse, the mismatched impedance along the measurement chain (Figure 3(a)) causes a deterioration of the modulation efficiency, $m$, (Figure 3(b)). This detection chain temporal-response drove us to model the incident optical pulse by a gaussian with a full width at half maximum ( $F W H M$ ) equals to 390 ps. The modulation efficiency is defined by the following relation:

$$
m=\frac{A_{\max }-A_{\min }}{A_{\max }+A_{\min }} .
$$


where $A_{\max }$ is the mean of the pulse maximum amplitudes, and $A_{\min }$ is the mean of the pulse minimum amplitudes. The modulation efficiency was calculated from simulations where the modulation frequency, $F_{m}$, was varied from 1 to $3 \mathrm{GHz}$ (Figure 9). One should note the marked decrease of the modulation efficiency when $F_{m}$ is increasing.

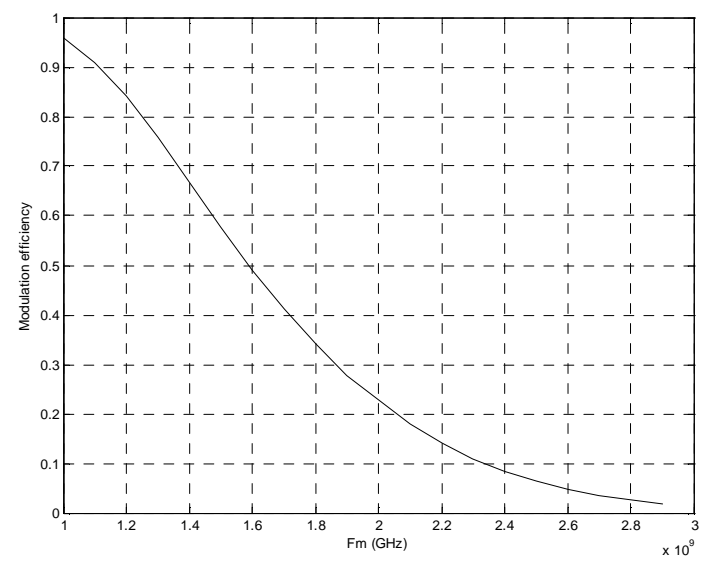

Figure 9. Modulation efficiency $m$ function of $F_{m}$

These results are consistent with experimental data, since for $F_{m}=1.5 \mathrm{GHz}$ the calculated modulation efficiency and the experimental one are alike (58\%). This $1.5-\mathrm{GHz}$ modulation frequency was, thus, kept to compare simulation results with experimental ones. In future experiments, efforts will be made to shorten the detection chain response decay time and consequently increase $m$. Another option is to decrease the modulation frequency, but it is detrimental to an accurate target ranging.

\subsection{A new modulation scheme}

According to the Fourier uncertainty relations, a long pulse train is more frequency selective than a short one, so the system performances should be greatly enhanced by a matched-filtering. However, a high number of pulses generates time-spread target echoes responsible for a deterioration of target ranging. Besides, in practice, such a configuration is at the origin of serious realization problems. Moreover, the adjustment of the optical delay line modulator system (section 2.2 ) in order to get a train composed of 4 accurately aligned pulses ( 7 liberty degrees) proved to be very difficult, which means that getting, for example, of a 16-pulse train will be nearly impossible, since 28 liberty degrees would be at play.

A very easily implemented technical solution to emit a long temporal pulse train with a reasonable loss of temporal shortness is the use of a Fabry-Perot resonator as modulator. This Fabry-Perot modulator (FP modulator) is composed of only 2 face-to-face optic elements, which drastically reduces the number of liberty degrees at play in alignment procedure (Figure 10). This geometry easily ensures intrinsic colinearity of light beams within the resonator and gives at the output a modulated train of decreasing-amplitude pulses. Moreover, the delay between two pulses is alike and equals to $\mathrm{v} / 2 L$ where $\mathrm{v}$ is the speed of light and $L$ is the resonator width. The number of pulses in a train depends on the output losses $(T)$ of the second mirror $\left(M_{2}\right)$, and thus a relevant choice of $T$ value enables one to get a very long train. 


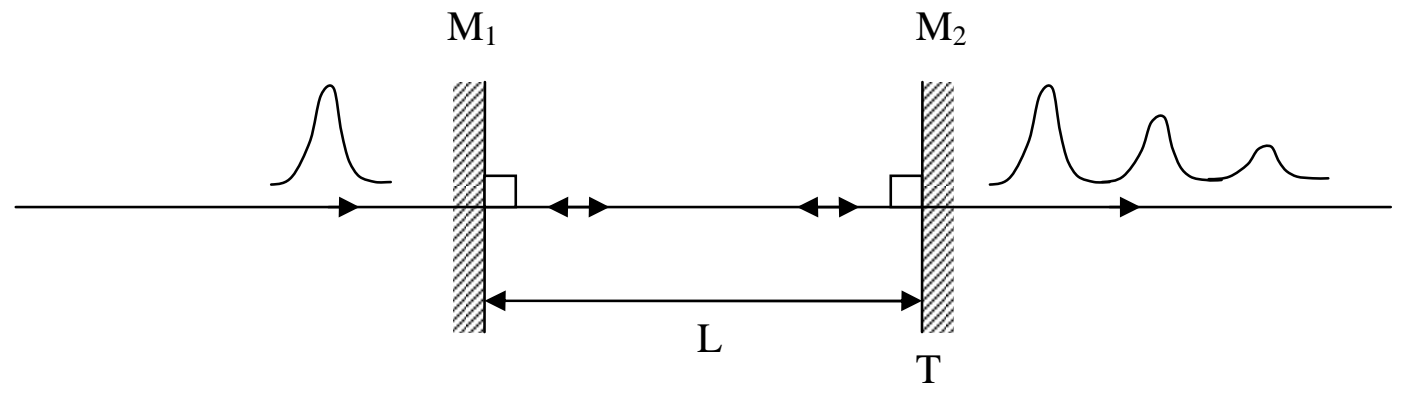

Figure 10. Fabry-Perrot resonator.

M1, M2: mirrors; L: resonator length; T: output losses of mirror $\mathrm{M}_{2}$

The intensity, $I_{n}$, of the $n^{\text {th }}$ pulse is given by:

$$
I_{n}=I_{0} \cdot\left[T(1-T)^{n}\right]
$$

where $I_{0}$ is the incident pulse intensity.

Simulations were run with this FP modulator to gain more insight into the impact by $T$ on the shape of the outpul signal and, thus, on the spectral selectivity. Figure 11 presents the simulated emitted signals for two different output coefficients $T=0.26$ and $T=0.12$ in the time and frequency domains (Figure 11(a) and Figure 11(b), respectively). It is worth recalling that the energy contained in each reference signal is alike (determined in the experimental reference signal $\left.E_{e}(t)\right)$. In both cases $(T=0.26$ and $T=0.12)$, the reference signal is composed of numerous pulses, but most of the energy is contained in the very first pulses. From the signal processing point of view, Figure 11(b) shows that the more numerous the pulses are, the thinner the spectrum is.

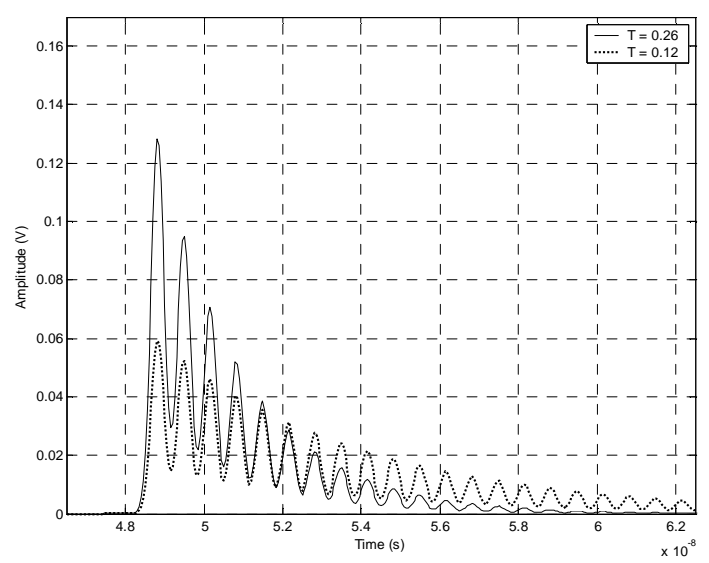

(a)

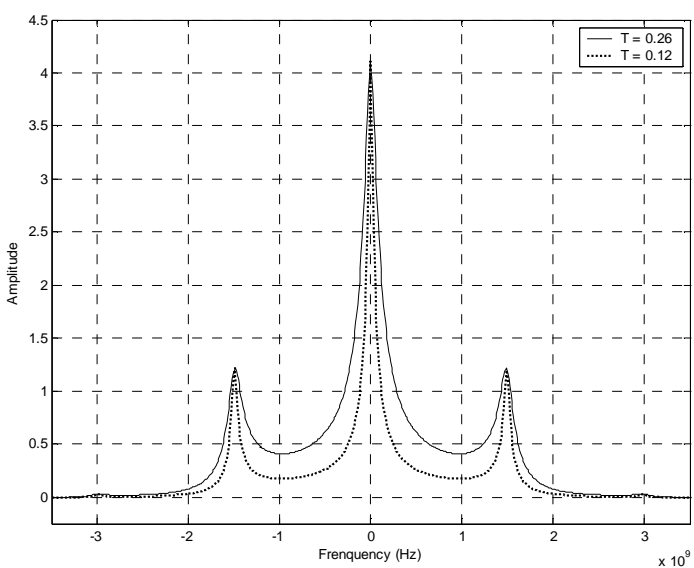

(b)

Figure 11. Reference signals emitted by FP modulators for $T=0.26$ and $T=0.12$;

(a) Time domain representation; (b) Frequency domain representation

According to the theory and in agreement with our simulations (Figure 12), when the number of pulses is increasing, the self-correlation gives wider $F W H M$, whereas the amplitude is decreasing. These features together with the easy production of long pulse trains make this new FP modulator very promising. 


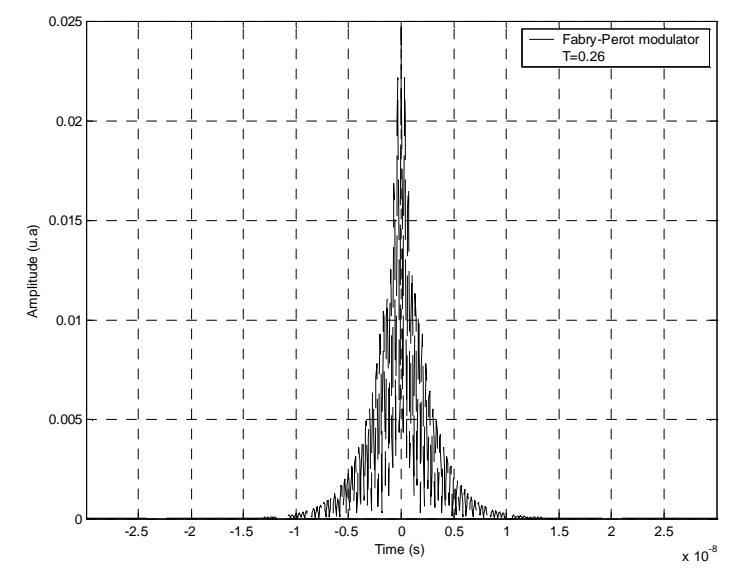

(a)

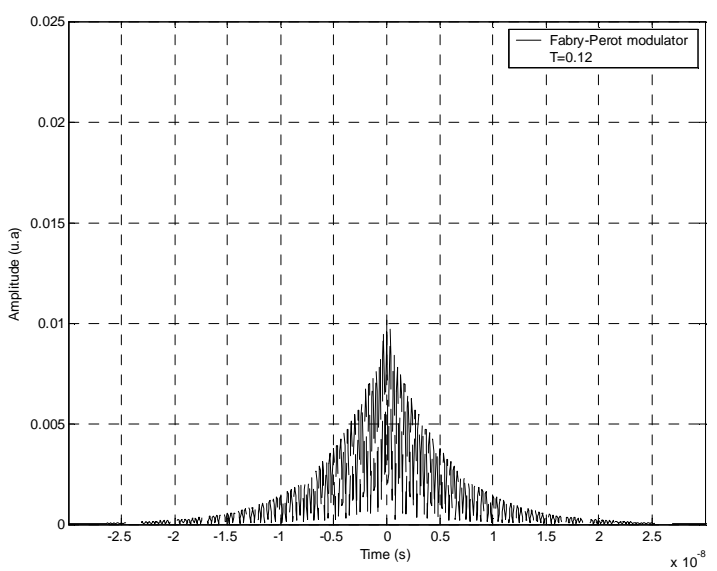

(b)

Figure 12. Results of self-correlation between reference signal and centered reference signal. (a) $T=0.26$; (b) $T=0.12$

\subsection{Variation of the emitted-train duration}

To compare the performances of the FP modulator with those of the delay line modulator, the modulation frequency was set at $1.5 \mathrm{GHz}$, and simulations of the backscattered signal, $S_{s}(t)$, were run for various emitted FP signals. Moreover, the value of the output losses, $T$, of the FP output mirror $\left(\mathrm{M}_{2}\right)$ was varied since this parameter governs the number of pulses in the reference signal and consequently the emitted-train duration (Figure 13). By convention, we set that only the pulses with an amplitude of, at least, $10 \%$ of the first pulse amplitude would be considered in the counting of the number of pulses contained in a given train.

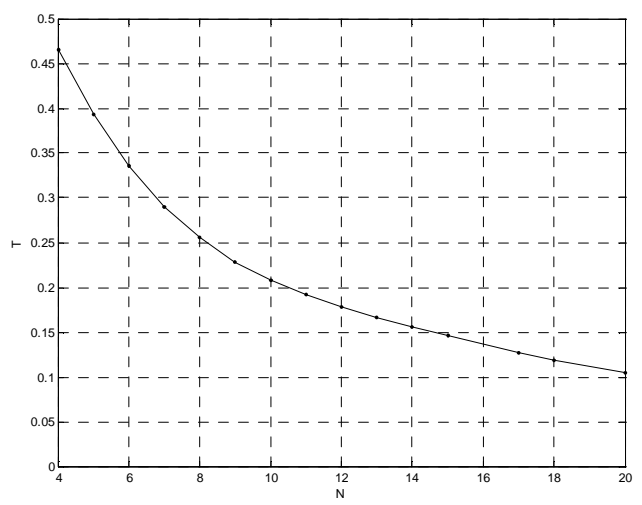

Figure 13. The value of $T$ determines reference signal pulse number $N$

After each injection of the reference signal into the realistic model, $M(t)$, and matched-filter processing, the performance criteria (SNR, SVBNR and Contrast) and FWHM of the processed target echo were calculated. Moreover, to simplify the simulated backscattering waveform, the set-up-induced spurious echoes were removed. Figure 14 presents the simulated backscattered signals and the processed signals for a pulse number equal to $N=8$ and $N=18$, i.e. $T=0.26$ and $T=0.12$, respectively. It shows that only 2 main echoes are still visible after matched-filtering. In agreement with the theory (Section 2.1), the water volume backscattering is nearly totally suppressed. After processing, SNR rises from 4.4 to 12.5 , for $\mathrm{N}=8$ and $\mathrm{N}=18$, respectively. The $S V B N R$ is also improved thanks to the filter noise rejection and reaches 1 for $\mathrm{N}=18$. 


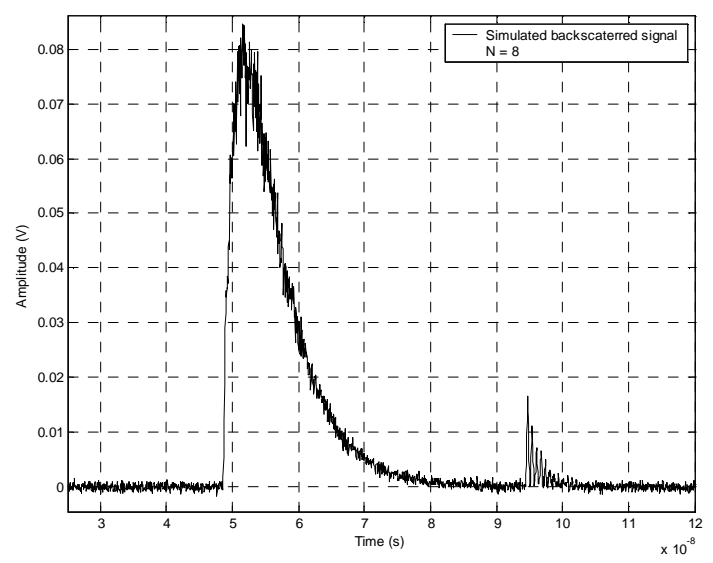

(a)

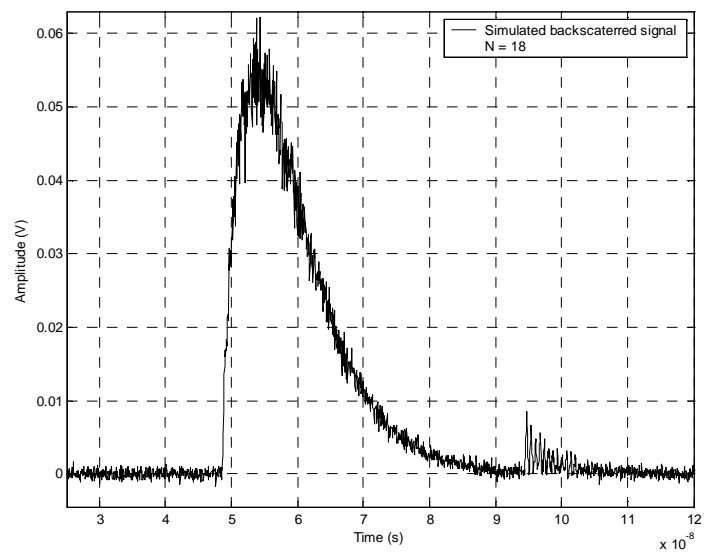

(c)

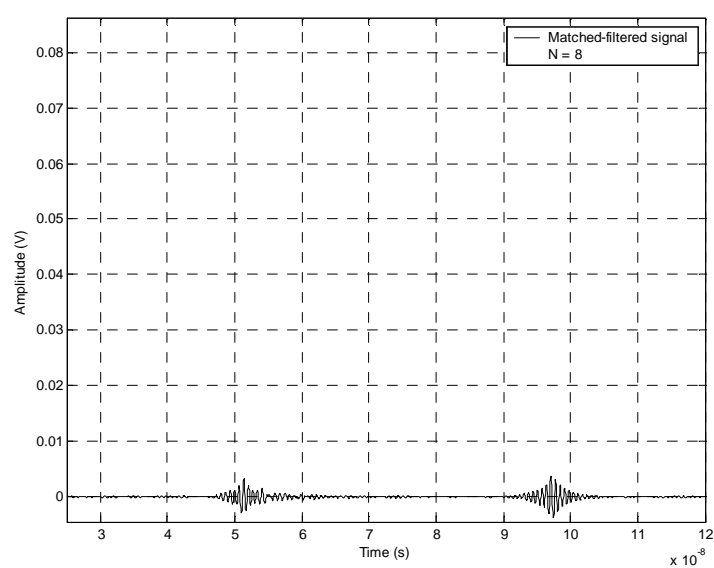

(b)

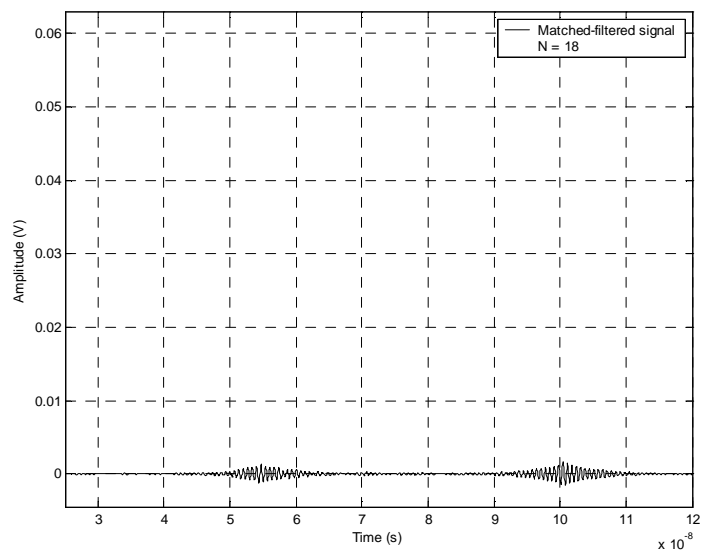

(d)

Figure 14. Simulated backscattered signal and matched-filtering results for $N=8$ and $N=18$ (i.e. $T=0.26$ and $T=0.12$, respectively)

The raw SNR (dash line Figure 15(a)) is unaffected by the number of pulses because the signal and noise energies are unchanged whatever the injected reference signal. On the other hand, the filtered-signal SNR (solid line) is substantially increasing with the number of pulses. Indeed, any increase of this number results in an enhancement of the matched-filter frequency selectivity and thus in a greater noise rejection. Theoretically, the higher the number of pulses is, the higher the $S N R$ is. However, theoretically the choice of a very high, $N$, would permit one to be certain of the presence of a target, but the target ranging would be not accurate.

The raw SVBNR (dash line Figure 15(b)) is also unaffected by the number of pulses for the reasons explained above for $S N R$. Conversely to the filtered-signal SNR, the filtered signal SVBNR (solid line Figure 15(b)) is increasing with the number of pulses. This result is of key interest because, theoretically, as soon as the modulation frequency, $F_{m}$, exceeds the water cut-off frequency, the water volume backscattering is suppressed, and the SVBNR is no longer filter selectivitydependent. However, matched-filtering not only reduces the water volume backscattering, but also rejects the white noise, which explains why $S V B N R$ is $N$-dependent.

For raw signals (dash line Figure 15(c)), any increase of the number of pulses causes a severe reduction of the contrast parameter. On the other hand, for filtered signals (solid line Figure 15(c)), an elevation of the number of pulses is accompanied with a slight reduction of contrast. Theses observations confirm the reduction of the signal background 
noise by the matched-filtering process whereas the target echo amplitude is less affected. As expected, any elevation of the number of pulses goes along with a marked increase of the $F W H M$ value (Figure 15(d)) of the processed target echo.

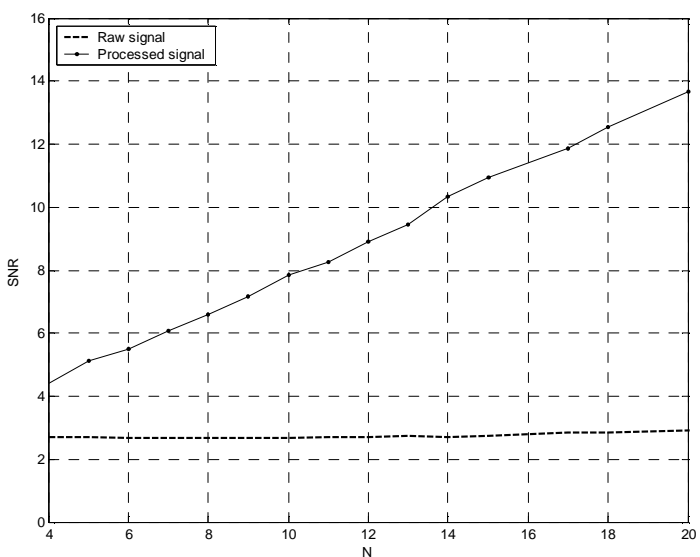

(a)

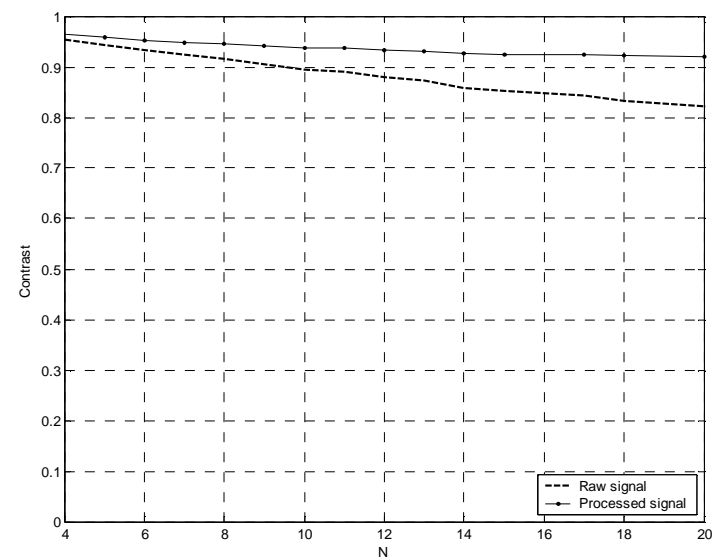

(c)

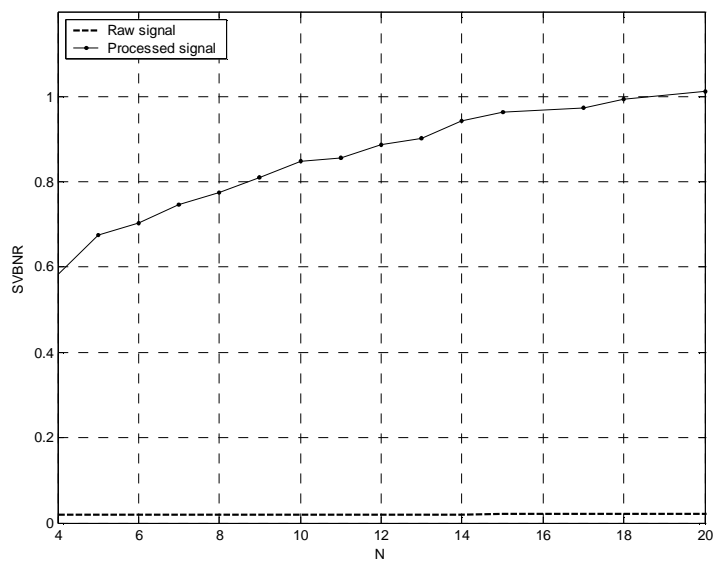

(b)

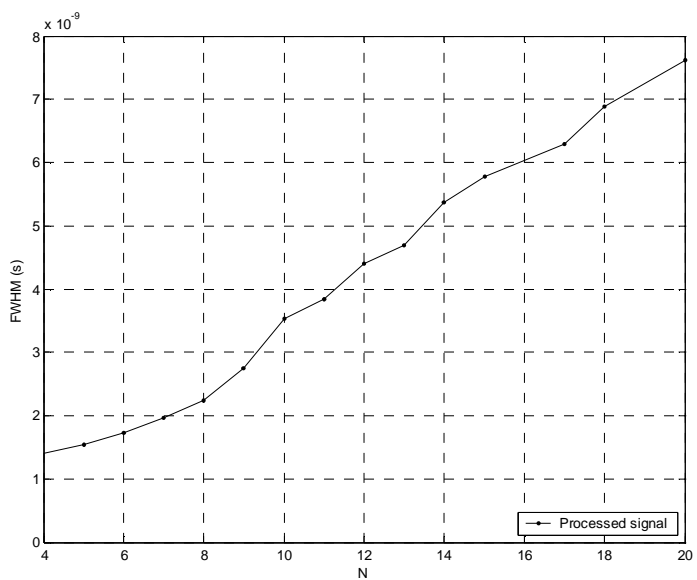

(d)

Figure 15. Simulations results as a function of the number of pulses

(a) SNR; (b) SVBNR; (a) Contrast; (b) Target echo FWHM

\subsection{Promising configurations}

The choice of the number of pulses depends on the detection configuration. In fact, if the aim is only to get evidence, or not, of the existence of a target, emission of long pulse trains is advised since the $S N R$ and $S V B N R$ are both higher. However, in the case where the goal is an accurate localization of the target (e.g. in-lab tank experiments or shallow target detection) emitting shorter pulse train is better. Finally, the choice of $\mathrm{N}$ value is ruled by the desired target ranging resolution. For example, for a shallow target immersed at a $1-\mathrm{m}$ depth, the maximum value for $N$ must not exceed $N=13$; if the target is at a $2-\mathrm{m}$ depth, this maximum value is $N=20$. 


\section{CONCLUSION}

A theoretical model was extracted from available experiments data and further used to simulate realistic backscattered signals for different input signals. Then, in order to develop a new modulator, an optimum set of amplitude modulation code parameters was extracted to maximize both the target signal-to-noise ratio and signal-to-volume-backscatteringnoise ratio. The Fabry-Perot resonator, with its specific geometry, gave conclusive and promising results. Simulations showed that the SNR and SVBNR are both greatly improved by emitting a long train of pulses. This is why when one wants to get evidence of the presence of a target, it is advised to emit a very long pulse train. On the other hand, to accurately locate the position of a target, the train has to be short. To conclude, the chosen number of pulses is a compromise between enhancement of both the SNR and SVBNR and target ranging-required resolution. On-going studies are focused on the realization of the new LIDAR set-up based on a Fabry-Perot resonator as modulator in order to carry out additional experiments in a 5-m long water tank.

\section{ACKNOWLEDGMENTS}

The authors thank Dr. M.P. Friocourt for help in the English manuscript preparation.This study was supported by DGA GESMA.

\section{REFERENCES}

[1] Cariou, J. and Lotrian, J., "Transmission characteristics of a pulsed laser beam in natural sea-water: determination of the attenuation coefficients in the 415-660 nm spectral range," J. Phys. D: Appl. Phys. 15, 1873-1880 (1982).

[2] Pellen, F. Intes, X. Olivard, P. Guern, Y. Cariou, J. Lotrian, J. "Determination of sea-water frequency response by backscattering transfer function measurement", J. Phys. D: Appl. Phys. 33, 349-354 (2000)

[3] Liang, J. Yang, K. Xia, M. and co, "Monte Carlo simulation for modulated pulse bathymetric light detecting and ranging systems", Journal of optics A: Pure and Applied Optics, 415-422, (2006).

[4] De Dominicis, L. Ferri de Collibus, M. Fornetti, G. Guarneri, M. Nuvoli, M. Ricci, R. Francucci, M. "Improving underwater imaging in an amplitude modulated laser system with radio frequency control technique", JEOS, Rapid Publications 5, 10004, (2010).

[5] Mullen, L. J. Vieira, A. J. C. Herczfeld, P. R. "Application of RADAR Technology to Aerial LIDAR Systems for Enhancement of Shallow Underwater Target Detection", IEEE Transactions on Microwave Theory and Techniques, vol. 43, $\mathrm{n}^{\circ}$ 9, (1995).

[6] Pellen, F. Olivard, P. Guern, Y. Cariou, J. Lotrian, J. "Radio frequency modulation on optical carrier for target detection enhancement in sea water", SPIE $46^{\text {th }}$ Annual Meeting on Optical Science and Technology, San Diego, (2001).

[7] Lotrian, J. Cariou, J. and Guern, Y. "Attenuation measurement in liquids by analysis of space-time structure of backscattered laser light pulses", Appl. Opt., vol. 29, N¹1, 1593-1594, (1990).

[8] Gordon, H. R. "Interpretation of airborne oceanic lidar: effects of multiple scattering", Appl. Opt., vol. 21, $\mathrm{N}^{\circ} 16,2996-3001,(1982)$.

[9] Poole, L. R. Venable, D. D. and Campbell, J. W. "Semianalytic Monte Carlo radiative transfer model for oceanographic lidar systems", Appl. Opt., vol. 20, N²0, 3653-3656, (1981). 